The Open Dentistry Journal
Bentham OPEN
CrossMark
Content list available at: www.benthamopen.com/TODENTJ/
DOI: $10.2174 / 1874210601610010236$

\title{
Orthodontic Treatment of Malocclusion and its Impact on Oral Health-Related Quality of Life
}

\author{
Abdolreza Jamilian ${ }^{1, *}$, Bita Kiaee ${ }^{2}$, Shabnam Sanayei ${ }^{2}$, Saeed Khosravi ${ }^{3}$ and Letizia Perillo ${ }^{4}$ \\ ${ }^{I}$ Department of Orthodontics, Tehran Dental Branch, Center of Craniofacial Research, Islamic Azad University, \\ Tehran, Iran \\ ${ }^{2}$ Departments of Orthodontics, Tehran Dental Branch, Islamic Azad University. Tehran, Iran \\ ${ }^{3}$ Department of Languages, Tehran University of Medical Sciences, Tehran, Iran \\ ${ }^{4}$ Departments of Orthodontics, Second University of Naples, Naples, Italy
}

Received: February 25, 2015

Revised: April 28, 2016

Accepted: April 28, 2016

\begin{abstract}
:
Objectives:

Malocclusion, though not life-threatening, has vast impact on individual's social interactions and self-esteem. Therefore, the aim of the current study was to assess whether orthodontic treatment of adolescents with malocclusion had any association with their oral health-related quality of life (OHRQoL).

\section{Methods:}

The subjects for this study were recruited at a state-funded university clinic. Data were collected from 100 participants aged 17 to 21 with moderate to severe malocclusion. Experimental group comprised of 50 subjects who were in the retention phase of their orthodontic treatment and the control group comprised of 50 untreated subjects. The shortened version of the Oral Health Impacts Profile (OHIP-14) as used to assess the subjects' oral health-related impact. T-test, Kruskal-Wallis, and Mann Whitney tests were used to analyze the data and $p$-value was set at $P<0.05$.
\end{abstract}

Results:

In general, oral health-related quality of life of all subjects significantly improved after orthodontic treatment. $(p<0.001)$ Subjects with moderate malocclusion showed better improvement than severe malocclusion subjects. $(P<0.001)$

\section{Conclusion:}

This study showed that oral health-related quality of life improves with the treatment of malocclusion.

Keywords: Malocclusion, OHIP, OHRQoL, Orthodontic treatment, Quality of life.

\section{INTRODUCTION}

Malocclusion in itself is not a life threatening condition; however, it may unfavorably affect social interactions and psychological well-being of patients [1 - 3].

Index of Orthodontic Treatment Need (IOTN) was proposed by Brook and Shaw [4] in the United Kingdom as a scoring system for estimating treatment need of patients with various degrees of malocclusion. The IOTN has been used extensively in the literature to evaluate actual and perceptive orthodontic treatment needs [5, 6]. Various studies have

\footnotetext{
* Address correspondence to this author at the No. 2713, Vali Asr St. Tehran 1966843133, Iran; Tel: 0098-21-22011892; Fax: 0098-21-22022215, E-mail: info@jamilian.net
} 
also evaluated IOTN and its impact on quality of life. Quality of life (QoL) has been defined by the World Health Organization as "people's perception of their position in life in the context of the culture and value systems in which they live and in relation to their goals, expectations, standards, and concerns". Additionally, oral health-related quality of life (OHRQoL) describes the impact of oral and paraoral conditions on one's life. Recognizing the effects of orthodontic treatment on the quality of life is an important step in informing the patients of the health benefits and gains of orthodontic treatment [7].

\section{Table 1. IOTN treatment grades.}

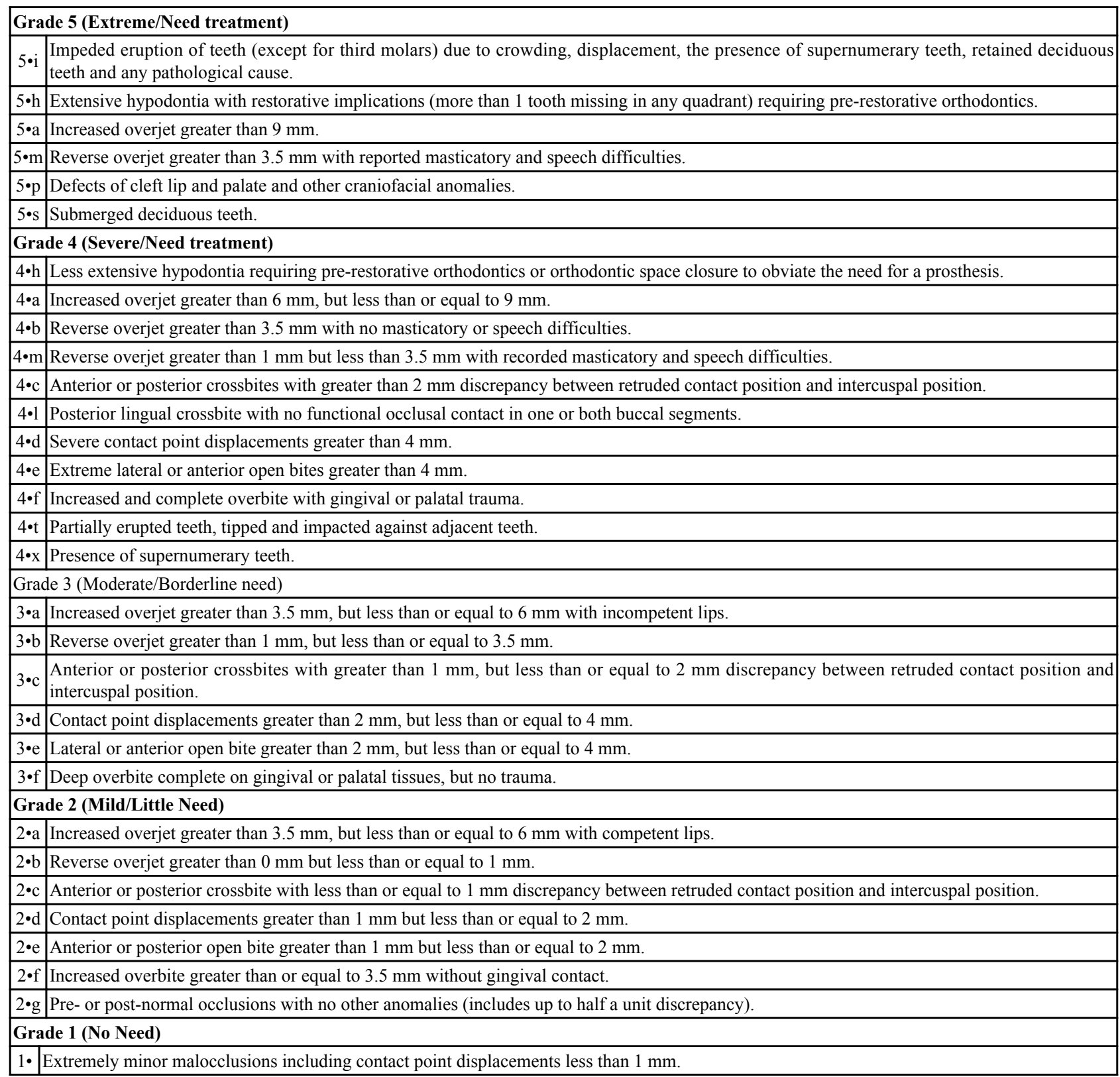

Several studies have investigated the effects of orthodontic treatment on OHRQoL between treated patients and untreated control groups [8]. de Oliveira and Sheiham studied the impact of orthodontic treatment on oral health-related quality of life in Brazilian adolescents and found better oral health-related quality of life in adolescents who had completed orthodontic treatment [9]. However, Taylor et al. [10] did not find any significant association between orthodontic treatment of malocclusion and oral health related quality of life despite the improvement in the health, appearance, social well-being and oral function of the patients. Johal et al. [11] also assessed the impact of orthodontic treatment on OHRQoL in adults and found that during the first 3 months of treatment fixed orthodontic therapy had a negative impact on the OHRQoL of the patients, which was followed by an 
improvement in comparison with the pre-treatment scores.

Given the disagreements in the literature and lack of proper data the aim of this study was to compare the effects of malocclusion and its orthodontic treatment on the quality of life of adolescents.

Table 2. Demographic characteristics of the subjects.

\begin{tabular}{|c|c|c|c|c|c|}
\hline \multirow{2}{*}{ Group } & Severity of Malocclusion & \multicolumn{2}{|c|}{ Gender } & Age \\
\cline { 2 - 6 } & IOTN Grade & Number & Male & Female & $19.96 \pm 4.1$ \\
\hline \multirow{2}{*}{ Experimental } & 3 (Moderate) & 25 & 8 & 17 & $19.2 \pm 3.64$ \\
\cline { 2 - 6 } & 4 (Severe) & 25 & 6 & 19 & $18.32 \pm 3.66$ \\
\hline \multirow{2}{*}{ Control } & 3 (Moderate) & 25 & 10 & 15 & $19.32 \pm 4.35$ \\
\cline { 2 - 6 } & 4 (Severe) & 25 & 9 & 16 & 0.5 \\
\hline
\end{tabular}

\section{MATERIALS AND METHODS}

This historical cohort study received approval from the Human Research Ethics Committee of Islamic Azad University of medical sciences (IAU). Prior to data collection, only the subjects who agreed to participate by signing a statement of informed consent were included in the study.

A sample size calculation was carried out using data of a study investigating effects of malocclusion and its orthodontic treatment on the quality of life of adolescents and other similar studies which used convenient clinical samples for their study [3, 10]. It was estimated that a sample size of 46 subjects was needed to demonstrate a significant change in OHRQoL, with an 80 per cent probability power at the $5 \%$ level of significance. Considering possible dropouts, the samples size was increased by 10\%; resulting in a final sample size of 50 participants.

Table 3. Scores of OHRQoL based on OHIP-14.

\begin{tabular}{|c|c|c|}
\hline Group & IOTN Grade & Mean scores \\
\hline \multirow{2}{*}{ Experimental } & 3 (Moderate) & $1.12 \pm 1.69$ \\
\cline { 2 - 3 } & 4 (Severe) & $3.88 \pm 3.22$ \\
\hline \multirow{2}{*}{ Control } & 3 (Moderate) & $8.25 \pm 3.88$ \\
\cline { 2 - 3 } & 4 (Severe) & $20.76 \pm 6.64$ \\
\hline \multicolumn{2}{|c|}{} & $P$ Value \\
\hline
\end{tabular}

The samples were selected from people who were referred to orthodontic department of IAU from February to August 2012. Inclusion criteria was moderate to severe malocclusion using Index of Orthodontic Treatment Need (IOTN) grades of 3 and 4 [4, 12], age range of 17 to 21 years, and having the same socioeconomic factors including sex, race, social class, and level of education. None of the patients had undergone orthognathic surgery. Due to the strict inclusion criteria the final sample consisted of 100 subjects. The experimental group consisted of 50 subjects who had undergone orthodontic treatment for 2 years for treatment of their moderate to severe malocclusion. The control group included 50 untreated patients who had moderate to severe malocclusions. In both groups 25 of the subjects had moderate malocclusion (IOTN=Grade 3 ) and 25 of them had severe malocclusion (IOTN=Grade 4). Table 1 shows IOTN grading used in the study and Table 2 depicts the demographic and occlusion characteristics of the samples.

Table 4. Two by two comparison of OHRQoL scores.

\begin{tabular}{|c|c|c|c|c|c|c|c|}
\hline $\begin{array}{c}\text { Grade 3 } \\
\text { Experimental }\end{array}$ & $\begin{array}{c}\text { Grades 4 } \\
\text { Experimental }\end{array}$ & Grade 3 Control & $\begin{array}{c}\text { Grades 4 } \\
\text { control }\end{array}$ & a \& b & a \& c & c \& d & b \& d \\
\hline $1.12 \pm 1.69^{\mathrm{a}}$ & $3.88 \pm 3.22^{\mathrm{b}}$ & $8.25 \pm 3.88^{\mathrm{c}}$ & $20.76 \pm 6.64^{\mathrm{d}}$ & 0.001 & 0.001 & 0.001 & 0.001 \\
\hline
\end{tabular}

The samples were asked to complete questionnaires incorporating the Short Form Oral Health Impact Profile (OHIP-14) [13 - 16], which is a 14-items questionnaire designed to measure self-reported functional limitation, discomfort and disability attributed to oral conditions. It is derived from an original extended version of 49-items [17]. The experimental group subjects filled out the forms two months after completion of their treatment in their retention phase and the control group subjects filled them out before the start of their treatment. Responses to the 14 items of OHIP were recorded in a 5 -point Likert scale $(0=$ never, $1=$ hardly ever, $2=$ occasionally, $3=$ fairly often, $4=$ very often). The scores ranged from 0 to 56. Therefore, higher OHIP-14 scores represent more negative effects of oral health on the quality of life. The Statistical Package for Social Sciences, Version 20 (SPSS Inc. Chicago, Illinois, USA) was 
used to analyze the data. T-test, Kruskal-Wallis, and Mann Whitney tests were used to analyze the data and p-value was set at $P<0.05$.

\section{RESULTS}

Table 2 shows that there were no statistically significant differences in gender, age, and severity of the malocclusions between the two groups. Subjects with moderate malocclusion who had undergone orthodontic treatment had the lowest mean score of $1.12 \pm 1.69$; while, the control group subjects with severe malocclusion had the highest mean score of $20.76 \pm 6.64$. Kruskal-Wallis test showed that mean scores of treated subjects were significantly lower than control group which is indicative of lower OHRQoL in subjects who haven't undergone orthodontic treatment $(P<0.001)$ (Table 3). However, in Table 4 Man-Whitney test shows that even after treatment subjects with sever malocclusion had lower OHRQoL than the subjects with moderate malocclusion.

\section{DISCUSSION}

OHIP-14 questionnaire showed that untreated moderate to severe malocclusions have a negative impact on the quality of life of adolescents.

This finding is consistent with previous studies, which indicate that Brazilian adolescents who had undergone orthodontic treatment had less physical, psychological, and social complications associated with malocclusion in comparison with those who had no history of orthodontics [18]. Silvola et al. [19] found that the treatment of severe malocclusion significantly improved oral health-related quality of life of the patients. Foster et al. [20] examined the relationship between clinical characteristics (such as caries experience and malocclusion) and oral health-related quality of life (OHRQoL) in adolescents in New Zealand and could not find any relationship between caries experience and OHRQoL, while they found that malocclusion directly affects OHRQoL. de Oliveira et al. [9] found that completion of orthodontic treatment had a positive impact on the oral health-related quality of life of adolescents. Nonetheless, quite interestingly, they found that orthodontic treatment may have negative impacts on quality of life during the treatment and suggested that orthodontists should be aware of this impact caused by treatment and regularly remind patients of the positive outcomes. On the other hand, Taylor et al. [10] found that malocclusion and orthodontic treatment do not have any measurable effects on the OHRQoL. They mentioned that an explanation of their different finding could be what psychologists call a "hedonic treadmill" [21]. According to their study many believe that well-being has a fixed set point for each person that cannot easily be changed. And because orthodontic treatment takes place over several years, patients might have forgotten their initial condition and cannot evaluate their progress, or they might have already adapted to the final occlusal and esthetic outcome and, thus, there is no impact on OHRQoL. Therefore, changes in psychological measures after orthodontic treatment should not be expected [10].

On the contrary, our study showed that oral health-related quality of life improves as the occlusion is improved. Nonetheless, it should be noted that subjects with severe malocclusion showed lower OHRQoL improvement after orthodontic treatment in comparison with more moderate subjects. The reason for this might lie in the fact that although these subjects rejected surgery they still had the assumption that they would have benefited more from surgical correction.

One of the limitations to this study was that were no pretreatment OHRQoL assessments of treated subjects and longitudinal data on pre and post treatment could improve the results of the study.

\section{CONCLUSION}

Subjects with moderate to severe malocclusion with no history of orthodontic treatment had more negative OHRQoL than adolescents who had completed orthodontic treatment.

\section{CONFLICT OF INTEREST}

The authors confirm that this article content has no conflict of interest.

\section{ACKNOWLEDGEMENTS}

Declared none. 


\section{REFERENCES}

[1] Mohlin B, al-Saadi E, Andrup L, Ekblom K. Orthodontics in 12-year old children. Demand, treatment motivating factors and treatment decisions. Swed Dent J 2002; 26(2): 89-98.

[PMID: 12462877]

[2] Perillo L, Esposito M, Contiello M, Lucchese A, Santini AC, Carotenuto M. Occlusal traits in developmental dyslexia: a preliminary study. Neuropsychiatr Dis Treat 2013; 9: 1231-7. [http://dx.doi.org/10.2147/NDT.S49985] [PMID: 24009421]

[3] Liu Z, McGrath C, Hägg U. The impact of malocclusion/orthodontic treatment need on the quality of life. A systematic review. Angle Orthod 2009; 79(3): 585-91.

[PMID: 19413386]

[4] Brook PH, Shaw WC. The development of an index of orthodontic treatment priority. Eur J Orthod 1989; 11(3): 309-20. [PMID: 2792220]

[5] Perillo L, Masucci C, Ferro F, Apicella D, Baccetti T. Prevalence of orthodontic treatment need in southern Italian schoolchildren. Eur J Orthod 2010; 32(1): 49-53.

[http://dx.doi.org/10.1093/ejo/cjp050] [PMID: 19706641]

[6] Jamilian A, Darnahal A, Damani E, Talaeipour M, Kamali Z. Prevalence of orthodontic treatment need and occlusal traits in school children. Int Scholar Res Not 2014; 2014: 5. (Article ID 349793) [http://dx.doi.org/10.1155/2014/349793]

[7] Sergl HG, Klages U, Zentner A. Pain and discomfort during orthodontic treatment: causative factors and effects on compliance. Am J Orthod Dentofacial Orthop 1998; 114(6): 684-91. [http://dx.doi.org/10.1016/S0889-5406(98)70201-X] [PMID: 9844209]

[8] Perillo L, Esposito M, Caprioglio A, Attanasio S, Santini AC, Carotenuto M. Orthodontic treatment need for adolescents in the Campania region: the malocclusion impact on self-concept. Patient Prefer Adherence 2014; 8: 353-9.

[PMID: 24672229]

[9] de Oliveira CM, Sheiham A. Orthodontic treatment and its impact on oral health-related quality of life in Brazilian adolescents. J Orthod 2004; 31(1): 20-7. [http://dx.doi.org/10.1179/146531204225011364] [PMID: 15071148]

[10] Taylor KR, Kiyak A, Huang GJ, Greenlee GM, Jolley CJ, King GJ. Effects of malocclusion and its treatment on the quality of life of adolescents. Am J Orthod Dentofacial Orthop 2009; 136(3): 382-92. [http://dx.doi.org/10.1016/j.ajodo.2008.04.022] [PMID: 19732673]

[11] Johal A, Alyaqoobi I, Patel R, Cox S. The impact of orthodontic treatment on quality of life and self-esteem in adult patients. Eur J Orthod 2014; 37(3): 233-7.

[http://dx.doi.org/10.1093/ejo/cju047] [PMID: 25214505]

[12] Jamilian A, Toliat M, Etezad S. Prevalence of malocclusion and index of orthodontic treatment need in children in Tehran. Oral Health Prev Dent 2010; 8(4): 339-43. [PMID: 21180670]

[13] Slade GD. Derivation and validation of a short-form oral health impact profile. Comm Dent Oral Epidemiol 1997; 25(4): $284-90$. [http://dx.doi.org/10.1111/j.1600-0528.1997.tb00941.x] [PMID: 9332805]

[14] Ravaghi V, Farrahi-Avval N, Locker D, Underwood M. Validation of the persian short version of the oral health impact profile (OHIP-14). Oral Health Prev Dent 2010; 8(3): 229-35 [PMID: 20848000]

[15] Cicciù M, Matacena G, Signorino F, Brugaletta A, Cicciù A, Bramanti E. Relationship between oral health and its impact on the quality life of Alzheimer's disease patients: a supportive care trial. Int J Clin Exp Med 2013; 6(9): 766-72. [PMID: 24179569]

[16] Bramanti E, Matacena G, Cecchetti F, Arcuri C, Cicciù M. Oral health-related quality of life in partially edentulous patients before and after implant therapy: a 2-year longitudinal study. Oral Implantol (Rome) 2013; 6(2): 37-42. [PMID: 24175052]

[17] Slade GD, Spencer AJ. Development and evaluation of the Oral Health Impact Profile. Comm Dent Health 1994; $11(1)$ : 3-11. [PMID: 8193981]

[18] Bernabé E, Sheiham A, Tsakos G, Messias de Oliveira C. The impact of orthodontic treatment on the quality of life in adolescents: a casecontrol study. Eur J Orthod 2008; 30(5): 515-20. [http://dx.doi.org/10.1093/ejo/cjn026] [PMID: 18753305]

[19] Silvola AS, Rusanen J, Tolvanen M, Pirttiniemi P, Lahti S. Occlusal characteristics and quality of life before and after treatment of severe malocclusion. Eur J Orthod 2012; 34(6): 704-9. [http://dx.doi.org/10.1093/ejo/cjr085] [PMID: 21750239]

[20] Foster Page LA, Thomson WM, Ukra A, Baker SR. Clinical status in adolescents: is its impact on oral health-related quality of life influenced by psychological characteristics? Eur J Oral Sci 2013; 121(3 Pt 1): 182-7. 
[http://dx.doi.org/10.1111/eos.12034] [PMID: 23659241]

[21] Diener E, Lucas RE, Scollon CN. Beyond the hedonic treadmill: revising the adaptation theory of well-being. Am Psychol 2006; 61(4): 305-14.

[http://dx.doi.org/10.1037/0003-066X.61.4.305] [PMID: 16719675]

(C) Jamilian et al.; Licensee Bentham Open.

This is an open access article licensed under the terms of the Creative Commons Attribution-Non-Commercial 4.0 International Public License (CC BY-NC 4.0) (https://creativecommons.org/licenses/by-nc/4.0/legalcode), which permits unrestricted, non-commercial use, distribution and reproduction in any medium, provided the work is properly cited. 